\title{
Beyond Cancer Stem Cells: Understanding Cancer Heterogeneity Through Gene Regulatory Networks and Single Cell Analysis
}

\author{
Ivan Gomez ${ }^{1 \#}$, Mitra Mojtahedi" ${ }^{1 \#}$, Wei Wu${ }^{1 *}$ and Wei Cao ${ }^{2 *}$
}

${ }^{1}$ Institute for Biocomplexity and Informatics, Department of Bio Sciences, University of Calgary, Canada

${ }^{2}$ Clinical research center, affiliated people's hospital of Zhengzhou, South medical University, 33 Yellow River Road, Zhengzhou, Henan province, 45003 China

\#Contributed equally to the manuscript

\begin{abstract}
Cancer is a genetic and developmental disorder. The recent advents of systematic sequencing studies have revealed thousands of somatic mutations, hundreds of chromosome rearrangements and copy number variants in the cancer genomes. The genomic changes can differ markedly between individual cancers, even within a particular cancer type, individual tumours often display wide variations. Heterogeneity of cancer or cancer cells is the well observed phenomenon and is considered the major hurdle for successful treatment. Mutational clonal evolution and cancer stem cell models could only partially explain the ubiquitous heterogeneous tumour cell population. Here we provide intuitive thinking with systems biology view to explain the inevitable heterogeneity of cancer cells as an abnormal cell type driven by dynamics of gene regulatory network. We also discuss the significance of the single cell transcriptome for detecting the heterogeneous cancer cells and targeted therapy.
\end{abstract}

Keywords: Cancer stem cells (CSC); Gene regulatory networks(GRN); Heterogeneity; Stochasticity; Gene expression profiling

\section{Introduction}

Cancer is a systematic disease with the influence of genetic, epigenetic alterations and the interaction with macro- and micro-environment [1]. From the observation of histopathologic hallmarker of cancer cells to the discovery of aberrant chromosome changes in cancer cells, from the identification of oncogenes and tumour suppressor genes to the exploration of unique gene expression profiling of cancer or its subtype, and from the sequencing of exomic cancer genes to decoding the whole cancer genome, each milestone of cancer research over a century significantly deepens our understanding of cancer biology and change our view of the landscape of cancer genomics [2]. We have understood much more the molecular mechanisms of cancer development and complicated pathways operate in cancer cells and developed targeted therapies for certain type of cancers [1]. However, the heterogeneity of cancer cells leading to various response to treatment, recurrence and relapse after initial treatment is recognized the major hurdles to cure cancers [3]. Clonal evolution of mutation model assumes that random mutation and natural selection over time creates the heterogeneous phenotype. Cancer stem cell hypothesis conceives that differentiation from stem like cancer cells is responsible for the heterogeneous population [4]. Recently, the momentum of systems biology in cancer research provide a holistic thinking of cancer development with gene regulatory networks [5], The integration of the cancer stem cells (CSC, or tumour initiating cells) hypothesis and cell attractor theory with gene regulatory network may provide comprehensive insight of cancerous heterogeneity and potential therapeutic strategy. Here, we will discuss the cancer stem cell hypothesis, the concept of cell attractor and gene regulatory network, and the examination of single cell transcriptome instead of population to dissect heterogeneous tumour cell population for targeted therapy.

\section{Cancer stem cell hypothesis}

In 1993, CSCs were first identified in haematological malignancies [6], later their existence have been proved in various solid tumours [7]. CSC model proposes that a small proportion of cancer cell gives rise to a hierarchy of cells that proliferate and differentiate and contributes to the heterogeneity [8]. It is now clear that various tumours can be sustained by CSCs, which share some of the defining properties of normal stem cells such as self-renewal, expansion and multipotency. CSCs are also able to spontaneously or induceably differentiate to multiple different lineages of cancer cells that comprise the heterogeneous tumour cells [9]. Notably, CSCs can escape conventional therapy to regenerate a tumour, therefore, CSCs are accused for the culprit of the failure of treatment [6]. Certainly, specifically targeting CSCs will ideally improve the therapeutic efficacy [10]. However, several studies have reported that non-cancer stem cells can convert into acquiring cancer stem-cell-like behaviour and supporting tumour formation [10-13]. We reason that cell type switch in tumour cells is dynamic and different cell type co-exist seems to be necessary to maintain their survival. The complexity of cancer biology promote us to revisit the cell attractor and gene regulatory networks which govern cell fate proposed by Stuart Kauffman in 1960's [12] that could help to understand the heterogeneity of cancer cells in addition of cancer stem cell model and will be discussed briefly on the next section.

The concept of cell types as attractors on gene regulatory networks and the inevitability of cancer

Cell types in general are discrete, mutually exclusive and stable

${ }^{*}$ Corresponding author: Wei Wu, Institute for Biocomplexity and Informatics Room \# 547, Biological Sciences Building, 2500 University Dr. NW Calgary, Alberta, CanadaT2N 1N4, Tel: 403-220-3266; Fax: 403-210-8655; E-mail: wuwei@ ucalgary.ca

Wei Cao, Clinical research center, affiliated people's hospital of Zhengzhou, South medical University, 33 Yellow River Road, Zhengzhou, Henan province, 45003 China, Tel: 0118637167077619; E-mail: caoweiyu@hotmail.com

Received October 15, 2011; Accepted November 13, 2011; Published Novembe 15,2011

Citation: Gomez I, Mojtahedi M, Wu W, Cao W (2011) Beyond Cancer Stem Cells: Understanding Cancer Heterogeneity Through Gene Regulatory Networks and Single Cell Analysis. J Stem Cell Res Ther S7:002. doi:10.4172/2157-7633.S7-002

Copyright: (C) $2011 \mathrm{Gomez} \mathrm{I,} \mathrm{et} \mathrm{al.} \mathrm{This} \mathrm{is} \mathrm{an} \mathrm{open-access} \mathrm{article} \mathrm{distributed} \mathrm{unde}$ the terms of the Creative Commons Attribution License, which permits unrestricted use, distribution, and reproduction in any medium, provided the original author and source are credited. 
phenotypic states. These distinct cellular states include immature stem and progenitor cells as well as the fully differentiated mature cells. Each of them is characterized by a unique genetic circuit which we can now approximately measure with specific gene expression program [13]. Regarding the origin and induction of cancerous cells from normal cells we could consider two hypotheses. One is that cancer origin is "cell-intrinsic", according to which it arises by the modification of a pre-existing intra-cellular program which is highly influenced by the stochastic change on gene networks [14]. The other is assuming that a "deterministic" process in which cancer is caused by instructive changes (DNA mutations and/or epigenetic modifications) which precisely induce genetic program modifications in order to generate a new (malignant) phenotype [15]. A unification of these two phenomenological hypotheses is possible when we look at cancer origin through the lenses of non-linear dynamics of a molecular interaction network, or more precisely, a Gene Regulatory Network (GRN). This GRN determines the expression levels of the $N$-genes that it encompasses at a given time point (for a clean and deep introduction to GRN dynamics see $[16,17])$.

In this network, the connection and interdependence between $\mathrm{N}$ regulatory molecular elements establish a $N$-dimensional system whose dynamics control the trajectory of the cell differentiation process. In a mathematical sense, the cell moves in an $\mathrm{N}$-dimensional "state space" in which each point represents a particular gene expression profile [13]. The regulatory interactions now impose heavy constraints on how the expression levels of individual genes can change, as a consequence, it maps mathematically in the highly constrained way in which the cell, or the point in the state space, can move: it moves only along predestined trajectories. This is the integrated dynamics of the network [17].

A trajectory is not only determined by the nature of the interactions but is highly influenced by gene expression noise, which confers certain stochasticity to the course of the trajectory. However, the system dynamics are not totally erratic due to the presence of well established interactions that keep the trajectories towards stable points (attractors). Usually GRN is composed of gene circuits of mutually repressing genes (here the repression is a constraining interaction) implies a wellordered and predictable dynamics, resulting in particular trajectories where several stable steady states are observed to which the system (point in the state space representing the cell) is "attracted". This sense of directionality in the system typically starts a binary decision of the undecided state and ends in the state of the committed lineage [18].

At this point we could observe gene mutations as physical modifications on the gene network architecture and, as we know that one gene expression usually maps into one phenotype, gene mutations and epigenetic changes can be accused for the origin of new malignant phenotypes (Figure 1). Basically, these changes are the ones who motivate the exploration of new viable phenotypes and create a side path that could be malignant-an unusual attractor [17]. The gene regulatory instability promotes further explorations and at the same time results in increasing the phenotypical heterogeneity of tumours and promoting the emergence of some cancer cells with stem cell like behavior, being those cell states immature and able to generate an entire hierarchic differentiation tree [6].

Under this perspective we could infer that: 1) cancer origin is a multifactorial phenomenon that may depend on genetic and nongenetic changes, importantly, these changes influence the dynamics of a GRN which maps into a given particular phenotype; 2) cancer is inevitable and is a side event of the ability of organisms to visit unused

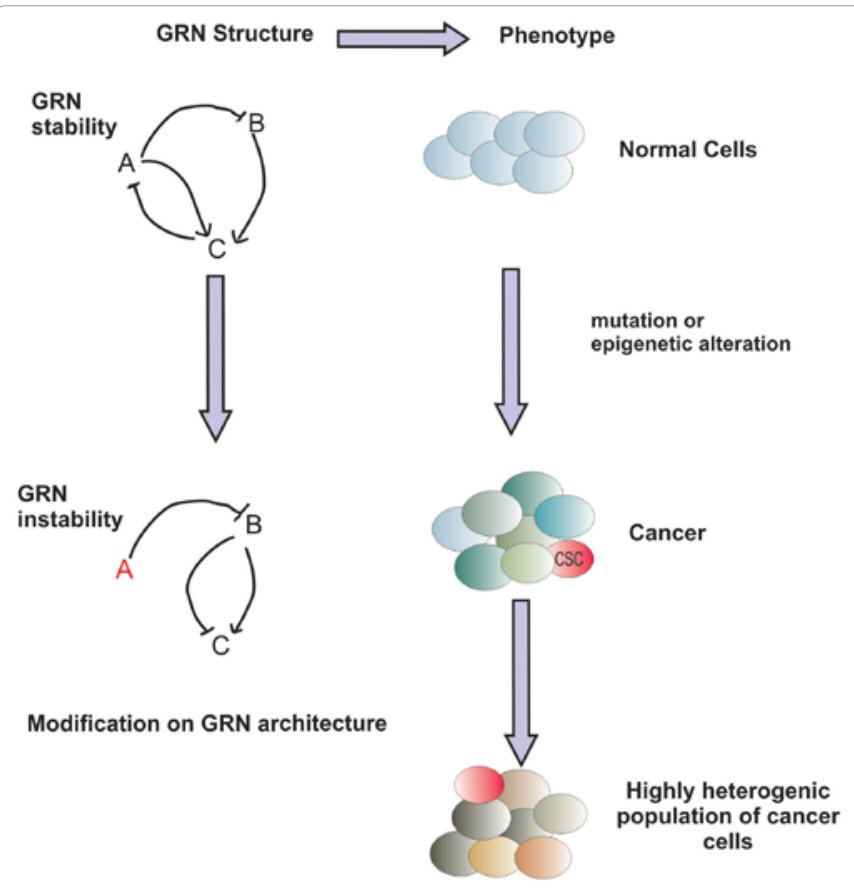

Figure 1: Changes on the genome such as mutations or epigenetic modifications induce Gene Regulatory Network (GRN) instability. This is due to modifications on the GRN architecture which produce highly heterogeneous populations and promote the exploration of new and viable phenotypes like Cancer Stem Cells (CSC).

states-malignant phenotype; 3) cancerous phenotypical heterogeneity is important for the development of tumours, making possible the appearance of cells with a stem cell profile. The last fact will be discussed further below.

\section{Heterogeneity in tumour cell populations}

Traditionally, cell population with particular traits presents in a bell shape distribution when measured by flow cytometry, this type of variation (the deviation from the mean) is termed micro-heterogeneity [19] which is determined by GRN. Tumour heterogeneity may be categorized into inter-tumoural and intra-tumoural heterogeneity with respect to both function (such as response to treatment) and phenotype. The former refers to the variability among tumours in the same organ. For an example, breast cancer is classified five subtypes: luminal A, luminal B, basal-like, ERBB2/HER2 ${ }^{+}$and normal breast like, each subtype has a unique morphology, gene expression profiling and specific treatment [20]. Cell origin and genetic alterations may be the main reason resulting in the inter-tumoral variation [21]. Intratumoral heterogeneity defines as the variation within a tumour with respect to both functional properties and expression of markers. Hierarchical cancer stem cell and stochastic clonal evolution models are mainly taken into account to describe intra-tumoural heterogeneity phenomenon [16]. Stochastic clonal evolution model argues that genetic and epigenetic changes in cancer cells might give rise to other cancer cells with different phenotypes [22]; While cancer stem cell model assumes a sub-population of cells in a tumour proliferate and differentiate into different cells [4]. Individual cells within a population of cancer cells can be different genetically and epigenetically. Genetic heterogeneity refers to the differences among the genome sequence of the members of the population that result in a heterogeneous 
population [19]. On the other hand, non-genetic heterogeneity refers to the differences among individuals due to variability in expression level of the genes [23]. In the state space, the individual cancer cells positions at different attractors with stable GRN. Consistent with this idea, experimentally sorting out two subpopulation: cells with $\mathrm{CD} 44^{+}$ or $\mathrm{CD} 24^{+}$, in breast cancer, each subpopulation displays different gene expression profiles and different SNP pattern [24].

Cellular and molecular mechanisms underlying heterogeneity of tumours have remained one of the interesting and unanswered questions in the field of cancer biology. The cancer cell population diversity (intratumour heterogeneity) is of great importance to investigate due to its profound implications for disease progression, diagnosis and treatment [25]. In order to precisely identify cancer cells or cancer stem cells within a heterogeneous tumour population, we need to find their molecular signatures through performing gene expression analysis. For example, Shipitsin et al. [24] has been shown a differential expression of genes involved in TGF- $\beta$ pathway in $\mathrm{CD}_{4} 4^{+}$and $\mathrm{CD} 24^{+}$subpopulation cells, therefore, eliminating CD $44^{+}$ subpopulation (cancer stem cells) through inhibiting TGF-ß signaling becomes effective treatment [24]. To achieve such stratification of subpopulation in heterogeneous tumor cells, a unique technique, single cell gene expression detection is necessary to be developed.

\section{Gene expression profiling (GEP) of individual cancer cells and its significance in cancer biology}

Gene expression profile is a pattern of expression of thousand of genes across genome at a particular time under particular condition [23]. Gene expression profiles or patterns are generated by underlying gene regulatory network that defines and orchestrates the expression of genes across the genome. Each gene expression profile represents a state of the network [23] and therefore a cell type including cancer cell or cancer stem cell phenotypes. Gene expression profile also conveys information about how genes interact with each other and regulates each other's expression within a network [23].

Some techniques such as microarray, qPCR and RNA-sequencing (RNA-seq) have been used to measure gene expression profiles of population of cells. GEP using microarray has been done quite a lot in breast cancer to identify the genes associated with cancer and their level of activity at different stages of the disease [19-21]. Although systematic analysis of genome-wide transcription of cancer cells using microarray has offered not only revealing gene expression signature of the cells but also identifying biomarkers and therapeutic targets [26]. But the problem arises when we are aware that every cell within a population has its own gene expression profile and cell heterogeneity within a tumour is a common phenomenon [4]. This heterogeneity may arise from intrinsic stochastic gene expression in individual cell owing to different micro-environment or due to low copy number of transcriptional and translational molecules [27]. Techniques such as microarray that use population of cells as input in fact represent an average of gene expression profiles of all these cells [19]. Deciphering gene regulatory network underlying physiological phenotype, behaviour and function of cancer cells based on these data can be misleading and it masks the true gene expression pattern of individual cells [23]. Due to the presence of multiple subpopulations with different gene expression profiles, a more sensitive assay, ideally an assay at the single cell resolution is needed.

To be able to obtain these types of information, single cell microarray analysis [28] had been developed and could facilitate the analysis of evolution of tumour cells at the single cell resolution. Recent advances in technologies such as high-throughput (next-generation) sequencing technology, provides the opportunity to use individual single cells as starting input and investigate their transcriptomes [2123]. Although these techniques have been applied on single embryonic stem cells [29], or individual oocyte and blastomere cells [30] but they could be applicable to individual cancer cells as well. Identifying different subpopulations within a bulk of tumour to analyze the gene expression in single cancer cells could build the gene regulatory network at individual cell level and reveal such heterogeneity in very details. In addition, it can be used to develop targeted therapy and monitor the real-time response of cells to the treatment.

\section{Outlook}

Cancer is a genetic and developmental disorder. We have deeply understood the molecular mechanisms of cancer----about 400 cancer genes have been identified, hundreds of thousands of somatic mutations have been found, numerous chromosome rearrangements and few more novel fusion genes were unexpectedly uncovered in cancer genomes with the massively parallel sequencing technology [2]. Scientists are bewildered with piles of genomic data, and decoding cancer genome appears to be beyond the DNA sequencing. Reductionism encounters a bottleneck to answer the driving force of cancer and the origin of heterogeneity. The concept of systems biology may offer unique ideas to interpret the cancer cell as an abnormal cell type constitutively "instructed" by attractors and measured with gene regulatory network. Gene expression signature that is found for individual cells within a heterogeneous tumour could help us to specify subset of cancer cells within a heterogeneous population; with deep analysis of the pathways that are active in cancer stem cells we could target these cells specifically. In fact, Schatton et al. [31] has shown the first evidence in support of the potential therapeutic utility of CSCs in melanoma by demonstration that killing the CSC subpopulations selectively is enough to inhibit the growth of a tumour. These findings are promising for development of CSC-directed therapeutic strategies. In addition, cancer stem cell hypothesis reminiscent the attractor theory, induction of differentiation of cancer stem cell with small molecules appears to be feasible as an integrative treatment.

\section{References}

1. Hanahan D, Weinberg RA (2011) Hallmarks of cancer: the next generation Cell 144: 646-674.

2. Stratton MR, Campbell PJ, Futreal PA (2009) The cancer genome. Nature 458 719-724

3. Quintana E, Shackleton M, Foster HR, Fullen DR, Sabel MS, et al. (2010) Phenotypic Heterogeneity among Tumorigenic Melanoma Cells from Patients that Is Reversible and Not Hierarchically Organized. Cancer cell 18: 510-523.

4. Shackleton M, Quintana E, Fearon ER, Morrison SJ (2009) Heterogeneity in cancer: cancer stem cells versus clonal evolution. Cell 138: 822-829.

5. Huang S, Ingber DE (2007) A non-genetic basis for cancer progression and metastasis: self-organizing attractors in cell regulatory networks. Breast disease 26: 27-54

6. Wicha MS, Liu S, Dontu G (2006) Cancer stem cells: an old idea--a paradigm shift. Cancer research 66: 1883-1890; discussion 1895-1896.

7. Lorico A, Rappa G (2011) Phenotypic Heterogeneity of Breast Cancer Stem Cells. J Oncol 2011: 135039

8. Xu X, Qian H, Zhu W, Zhang X, Yan Y, et al. (2010) Isolation of cancer stem cells from transformed human mesenchymal stem cell line F6. Journal of molecular medicine 88: 1181-1190. 
Citation: Gomez I, Mojtahedi M, Wu W, Cao W (2011) Beyond Cancer Stem Cells: Understanding Cancer Heterogeneity Through Gene Regulatory Networks and Single Cell Analysis. J Stem Cell Res Ther S7:002. doi:10.4172/2157-7633.S7-002

9. Alison MR, Lim SML, Nicholson LJ (2011) Cancer stem cells: problems for therapy? J Pathol 223: 147-161.

10. Gupta PB, Onder TT, Jiang G, Tao K, Kuperwasser C, et al. (2009) Identification of Selective Inhibitors of Cancer Stem Cells by High-Throughput Screening. Cell 138: 645-659.

11. Chaffer CL, Brueckmann I, Scheel C, Kaestli AJ, Wiggins PA, et al. (2011) Normal and neoplastic nonstem cells can spontaneously convert to a stem-like state. Proc Natl Acad Sci U S A 108: 7950-7955.

12. Kauffman S (1971) Differentiation of malignant to benign cells. Journal of theoretical biology 31: 429-451.

13. Zhou JX, Huang S (2011) Understanding gene circuits at cell-fate branch points for rational cell reprogramming. Trends Genet 27: 55-62.

14. Huang S (2011) On the intrinsic inevitability of cancer: From foetal to fatal attraction. Semin cancer biol 21: 183-199.

15. Gilbertson RJ (2011) Mapping Cancer Origins. Cell 145: 25-29.

16. Huang S (2009) Non-genetic heterogeneity of cells in development: more than just noise. Development 136: 3853-3862.

17. Huang S, Ernberg I, Kauffman S (2009) Cancer attractors: a systems view of tumors from a gene network dynamics and developmental perspective. Seminars in cell \& developmental biology 20: 869-876.

18. Wang J, Xu L, Wang E, Huang S (2010) The potential landscape of genetic circuits imposes the arrow of time in stem cell differentiation. Biophysical journal 99: 29-39.

19. Huang S (2009) Non-genetic heterogeneity of cells in development: more than just noise. Development 136: 3853-3862.

20. Charafe-Jauffret E, Ginestier C, lovino F, Wicinski J, Cervera N, et al. (2009)
Breast cancer cell lines contain functional cancer stem cells with metastatic capacity and a distinct molecular signature. Cancer research 69: 1302-1313.

21. Visvader JE (2011) Cells of origin in cancer. Nature 469: 314-322.

22. Wang JCY, Dick JE (2005) Cancer stem cells: lessons from leukemia. Trends cell biol 15: 494-501.

23. Brock A, Chang $H$, Huang S (2009) Non-genetic heterogeneity--a mutationindependent driving force for the somatic evolution of tumours. Nature reviews. Genetics 10: 336-342.

24. Shipitsin M, Campbell LL, Argani P, Weremowicz S, Bloushtain-Qimron N, et al. (2007) Molecular definition of breast tumor heterogeneity. Cancer cell 11 259-273.

25. Botchkina IL, Rowehl RA, Rivadeneira DE, Karpeh MS, Crawford $\mathrm{H}$, et al (2009) Phenotypic subpopulations of metastatic colon cancer stem cells: genomic analysis. Cancer genomics \& proteomics 6: 19-29.

26. Quackenbush J (2006) Microarray analysis and tumor classification. The New England journal of medicine 354: 2463-2472.

27. Raj A, Oudenaarden AV (2008) Nature, nurture, or chance: stochastic gene expression and its consequences. Cell 135: 216-226.

28. Single-cell cDNA microarray profiling of complex biologica.pdf (n.d.).

29. Tang F, Barbacioru C, Wang Y, Nordman E, Lee C, et al. (2009) mRNA-Seq whole-transcriptome analysis of a single cell. Nat Methods 6: 377-382.

30. Tang F, Barbacioru C, Nordman E, Li B, Xu N, et al. (2010) RNA-Seq analysis to capture the transcriptome landscape of a single cell. Nat protoc 5: 516-535.

31. Schatton T, Murphy GF, Frank NY, Yamaura K, Waaga-Gasser AM, et al. (2008) Identification of cells initiating human melanomas. Nature 451: 345

This article was originally published in a special issue, Cancer Stem Cells handled by Editor(s). Dr. Vincent L. Cryns, Northwestern University, USA 